\title{
Weyl disks and square summable solutions for discrete symplectic systems with jointly varying endpoints
}

\author{
Roman Šimon Hilscher* and Petr Zemánek
}

"Correspondence:

hilscher@math.muni.cz Department of Mathematics and

Statistics, Faculty of Science, Masaryk University, Kotlárská 2,

Brno, 61137, Czech Republic

\begin{abstract}
In this paper we develop the spectral theory for discrete symplectic systems with general jointly varying endpoints. This theory includes a characterization of the eigenvalues, construction of the M-lambda function and Weyl disks, their matrix radii and centers, statements about the number of square summable solutions, and limit point or limit circle analysis. These results are new even in some particular cases, such as for the periodic and antiperiodic endpoints, or for discrete symplectic systems with special linear dependence on the spectral parameter. The method utilizes a new transformation to separated endpoints, which is simpler and more transparent than the one in the known literature.
\end{abstract}

MSC: Primary 39A12; secondary 34B20; 34B05; 47B39

Keywords: discrete symplectic system; eigenvalue; Weyl-Titchmarsh theory; $M$-lambda function; square summable solution; jointly varying endpoints; periodic endpoints

\section{Motivation}

In this paper we develop the spectral theory, in particular the Weyl-Titchmarsh theory, for discrete symplectic systems

$$
z_{k+1}(\lambda)=\left(\mathcal{S}_{k}+\lambda \mathcal{V}_{k}\right) z_{k}(\lambda)
$$

in which the dependence on the spectral parameter $\lambda \in \mathbb{C}$ is linear but other than that general. The term 'symplectic system' refers to the assumptions on the coefficients, which are complex $2 n \times 2 n$ matrices satisfying

$$
\begin{aligned}
& \mathcal{S}_{k}^{*} \mathcal{J} \mathcal{S}_{k}=\mathcal{J}, \quad \mathcal{S}_{k}^{*} \mathcal{J} \mathcal{V}_{k} \quad \text { is Hermitian, } \\
& \mathcal{V}_{k}^{*} \mathcal{J} \mathcal{V}_{k}=0, \quad \Psi_{k}:=\mathcal{J} \mathcal{V}_{k} \mathcal{J} \mathcal{S}_{k}^{*} \mathcal{J} \geq 0
\end{aligned}
$$

This implies that the coefficient matrix $\mathcal{S}_{k}+\lambda \mathcal{V}_{k}$ of $\left(\mathrm{S}_{\lambda}\right)$ and its fundamental matrix $\Phi_{k}(\lambda)$ satisfy the properties of symplectic matrices, i.e.,

$$
\left(\mathcal{S}_{k}+\lambda \mathcal{V}_{k}\right)^{*} \mathcal{J}\left(\mathcal{S}_{k}+\bar{\lambda} \mathcal{V}_{k}\right)=\mathcal{J}, \quad \Phi_{k}^{*}(\lambda) \mathcal{J} \Phi_{k}(\bar{\lambda})=\mathcal{J}
$$

(c) 2013 Šimon Hilscher and Zemánek; licensee Springer. This is an Open Access article distributed under the terms of the Creative Commons Attribution License (http://creativecommons.org/licenses/by/2.0), which permits unrestricted use, distribution, and reproduction in any medium, provided the original work is properly cited. 
Here $\mathcal{J}:=\left(\begin{array}{cc}0 & I \\ -I & 0\end{array}\right)$ is the canonical $2 n \times 2 n$ skew-symmetric matrix, the superscript $X^{*}$ denotes the complex conjugation, the matrices $\mathcal{S}_{k}$ and $\mathcal{J}$ are symplectic, and system $\left(\mathrm{S}_{\lambda}\right)$ can be viewed as a linear perturbation of the symplectic system $\left(\mathrm{S}_{0}\right)$, i.e., $z_{k+1}=\mathcal{S}_{k} z_{k}$. The principal aim of this paper is to study the problems with general jointly varying endpoints such as with the periodic endpoints $z_{0}=z_{N+1}$ or with the antiperiodic endpoints $z_{0}=-z_{N+1}$.

The theory of Weyl disks and square summable solutions for system $\left(\mathrm{S}_{\lambda}\right)$ was developed in the recent paper [1]. Earlier works dealt with special systems, in which the first $n$ equations do not depend on $\lambda$ (see [2-5]), i.e., for

$$
\mathcal{S}_{k}=\left(\begin{array}{cc}
\mathcal{A}_{k} & \mathcal{B}_{k} \\
\mathcal{C}_{k} & \mathcal{D}_{k}
\end{array}\right), \quad \mathcal{V}_{k}=\left(\begin{array}{cc}
0 & 0 \\
-\mathcal{W}_{k} \mathcal{A}_{k} & -\mathcal{W}_{k} \mathcal{B}_{k}
\end{array}\right), \quad \Psi_{k}=\left(\begin{array}{cc}
\mathcal{W}_{k} & 0 \\
0 & 0
\end{array}\right) \geq 0
$$

with Hermitian $\mathcal{W}_{k}$, or systems having a certain Hamiltonian structure (see [6, 7]). For a broader history of the Weyl-Titchmarsh theory for difference equations, we refer to $[1,8]$. The results in [4-7] have in common the following properties: (i) they are developed for the separated boundary conditions

$$
\alpha z_{0}=0, \quad \beta z_{N+1}=0, \quad \alpha, \beta \in \Gamma:=\left\{\alpha \in \mathbb{C}^{n \times 2 n}, \alpha \alpha^{*}=I, \alpha \mathcal{J} \alpha^{*}=0\right\},
$$

and (ii) they assume the so-called 'strong Atkinson condition', namely for some $N_{0} \in \mathbb{N}$,

$$
\sum_{k=0}^{N_{0}} z_{k+1}^{*}(\lambda) \Psi_{k} z_{k+1}(\lambda)>0
$$

for every nontrivial solution $z(\lambda)$ of $\left(\mathrm{S}_{\lambda}\right)$ on $[0, \infty)_{\mathbb{Z}}$ and every $\lambda \in \mathbb{C}$; see [9, Eq. (3.7.10)] and Hypotheses 2.1 and 2.2. The results in [1] are also derived for separated endpoints (1.3), but instead of (ii) the 'weak Atkinson condition' is required, namely for some $N_{0} \in \mathbb{N}$ inequality (1.4) holds only for every column $z(\lambda)$ of the natural conjoined basis $\tilde{Z}(\lambda)$ of $\left(\mathrm{S}_{\lambda}\right)$, which is defined as the solution starting with $\tilde{Z}_{0}(\lambda)=-\mathcal{J} \alpha^{*}$; see Hypotheses 4.1 and 4.2.

The change from the strong Atkinson condition to the weak Atkinson condition in [1] is crucial and absolutely essential, as it turns out in the present paper. Here we aim to extend the results in [1] to problems with general jointly varying endpoints

$$
\gamma\left(\begin{array}{c}
z_{0} \\
z_{N+1}
\end{array}\right)=0, \quad \gamma \in \Omega:=\left\{\gamma \in \mathbb{C}^{2 n \times 4 n}, \gamma \gamma^{*}=I, \gamma\left(\begin{array}{cc}
-\mathcal{J} & 0 \\
0 & \mathcal{J}
\end{array}\right) \gamma^{*}=0\right\} .
$$

The boundary conditions in (1.5) include, among others, the periodic endpoints $z_{0}=z_{N+1}$ or the antiperiodic endpoints $z_{0}=-z_{N+1}$, which could not be treated by the previous case in (1.3). The method we use is based on the augmentation of system $\left(\mathrm{S}_{\lambda}\right)$ into double dimension, which leads to a problem with separated endpoints having the original boundary conditions (1.5) as one of its constraints. This technique is known in the literature in principle ( $c f$. [10-15]), but the transformation to separated endpoints introduced in this paper is much simpler. At the same time, the transformed symplectic system no longer satisfies the corresponding strong Atkinson condition, but only its weak form. Thus, the derivation of the Weyl-Titchmarsh theory in [1] under the weak Atkinson condition is truly crucial for its further extension to jointly varying endpoints. 
For this general situation, we give a characterization of the eigenvalues of $\left(\mathrm{S}_{\lambda}\right)$ with (1.5), we construct the Weyl disks, their centers and matrix radii, and show the properties of square summable solutions. We also give an exact connection between the limit point or limit circle classification of the original system (in dimension $2 n$ ) and the augmented system (in dimension $4 n$ ). This connection reveals an interesting fact, namely that the limiting matrix radius of the augmented system has its rank at least $n$ (see Theorem 2.11), and so it is never zero in the limit point case as one would expect from the standard theory. The results of this paper (see Theorem 2.3) also imply the existence of multiple eigenvalues for scalar symplectic eigenvalue problems with jointly varying endpoints. This is known, e.g., for the second order discrete Sturm-Liouville problems with periodic endpoints in [16, Example 7.6] or [17, Theorem 2.2] and here we extend it to discrete symplectic systems. The new transformation of jointly varying endpoints into separated endpoints will also find applications in the continuous time problems or time scales problems (see, e.g., [15]). Finally, we remark that the results of this paper are new even for special discrete symplectic systems, such as those with (1.2), and also for the Jacobi equations (see [18]), symmetric three term recurrence equations (see [19-21]), and linear Hamiltonian difference systems (see $[6,22-24])$.

\section{Weyl-Titchmarsh theory for joint endpoints}

We start with some notation. By $I$ and 0 we denote the identity and zero matrices of a suitable dimension, which will be clear from the context. The discrete time intervals are denoted by $[a, b]_{\mathbb{Z}}:=[a, b] \cap \mathbb{Z}$, and similarly for other types of intervals. The Hermitian components of a square matrix $M$ will be denoted by $\operatorname{im}(M):=\left(M-M^{*}\right) /(2 i)$ and $\operatorname{re}(M):=$ $\left(M+M^{*}\right) / 2$. When $M$ is a scalar, then they reduce to the imaginary and real parts of the number $M$.

Let $\mathcal{S}_{k}, \mathcal{V}_{k}, \Psi_{k}$ be given $2 n \times 2 n$ matrices defined on $[0, \infty)_{\mathbb{Z}}$ and satisfying (1.1). Let $\gamma \in \Omega$ be a fixed $2 n \times 4 n$ matrix defining the boundary conditions in (1.5). With a given $N \in \mathbb{N}$, we consider the eigenvalue problem

$$
\left(\mathrm{S}_{\lambda}\right), \quad k \in[0, N]_{\mathbb{Z}}, \quad \lambda \in \mathbb{C}, \quad(1.5) .
$$

The eigenvalues of (2.1) are defined in a usual way. That is, $\lambda_{0} \in \mathbb{C}$ is an eigenvalue of (2.1) if system $\left(\mathrm{S}_{\lambda_{0}}\right)$ has a nontrivial solution $z$ on $[0, N+1]_{\mathbb{Z}}$ satisfying the boundary conditions in (1.5). In this case, $z$ is called an eigenfunction for $\lambda_{0}$ and the dimension of such eigenfunctions for $\lambda_{0}$ is its (geometric) multiplicity. As one of the main assumptions, we suppose that $\left(\mathrm{S}_{\lambda}\right)$ satisfies the strong Atkinson condition on a finite or infinite interval. An alternative terminology is that $\left(\mathrm{S}_{\lambda}\right)$ is definite on the given interval.

Hypothesis 2.1 (Strong Atkinson condition - finite) The inequality in (1.4) is satisfied with $N_{0}=N$ for every nontrivial solution $z(\lambda)$ of $\left(\mathrm{S}_{\lambda}\right)$ on $[0, N+1]_{\mathbb{Z}}$ and every $\lambda \in \mathbb{C}$.

Hypothesis 2.2 (Strong Atkinson condition - infinite) There exists $N_{0} \in \mathbb{N}$ such that inequality (1.4) holds for every nontrivial solution $z(\lambda)$ of $\left(\mathrm{S}_{\lambda}\right)$ on $[0, \infty)_{\mathbb{Z}}$ and every $\lambda \in \mathbb{C}$.

The results of this paper will be formulated with the aid of a particular fundamental matrix $\Phi(\lambda)$ of system $\left(\mathrm{S}_{\lambda}\right)$ starting with the initial value $\Phi_{0}(\lambda)=-\mathcal{J}$, i.e.,

$$
\Phi_{k+1}(\lambda)=\left(\mathcal{S}_{k}+\lambda \mathcal{V}_{k}\right) \Phi_{k}(\lambda), \quad k \in[0, \infty)_{\mathbb{Z}}, \quad \Phi_{0}(\lambda)=-\mathcal{J}, \quad \lambda \in \mathbb{C} .
$$


Our first result describes the orthogonality of the eigenfunctions and the multiplicity of the eigenvalues of (2.1). It generalizes [1, Theorem 2.8$]$ to jointly varying endpoints, compare also with [25, Theorem 2.2]. The proofs mostly follow by direct calculations in a similar way as the corresponding results in [1]. For completeness and comparison, we provide alternative proofs based on the transformation in Section 4 .

Theorem 2.3 Let $\gamma \in \Omega$ and $N \in \mathbb{N}$. Then the following statements hold.

(i) Under Hypothesis 2.1, the eigenvalues of (2.1) are real and the eigenfunctions corresponding to different eigenvalues are orthogonal with respect to the semi-inner product

$$
\langle z, \tilde{z}\rangle_{\Psi, N}:=\sum_{k=0}^{N} z_{k+1}^{*} \Psi_{k} \tilde{z}_{k+1}
$$

(ii) A number $\lambda \in \mathbb{C}$ is an eigenvalue of (2.1) if and only if the matrix

$$
L(\lambda):=\gamma\left(\begin{array}{c}
-\mathcal{J} \\
\Phi_{N+1}(\lambda)
\end{array}\right)
$$

is singular. In this case, the eigenfunctions corresponding to the eigenvalue $\lambda$ have the form $z=\Phi(\lambda) d$ on $[0, N+1]_{\mathbb{Z}}$ with a nonzero $d \in \operatorname{Ker} L(\lambda)$. Moreover, the geometric multiplicity of $\lambda$ is equal to its algebraic multiplicity, i.e., to $\operatorname{dim} \operatorname{Ker} L(\lambda)$.

Proof The statement follows from Theorem 4.3 with (4.6) and Corollary 4.4.

By Theorem 2.3, the multiplicities of the eigenvalues of (2.1) are at most $2 n$, compared to the separated endpoints case in [1, Theorem 2.8], in which the multiplicities of the eigenvalues are at most $n$. This implies that in the scalar case (i.e., for $n=1)$ there may exist multiple eigenvalues of (2.1). This phenomenon was observed in [16, Example 7.6] and later justified in [17, Theorem 2.2] for the periodic discrete Sturm-Liouville eigenvalue problem; see also Example 3.3.

Next we define the Weyl-Titchmarsh $M(\lambda)$-function for problem (2.1). For $k \in[0, N+1]_{\mathbb{Z}}$ and $\lambda \in \mathbb{C}$, we set

$$
M_{k}(\lambda):=-\left[\gamma\left(\begin{array}{c}
-\mathcal{J} \\
\Phi_{k}(\lambda)
\end{array}\right)\right]^{-1} \gamma\left(\begin{array}{c}
\mathcal{J} \\
\Phi_{k}(\lambda)
\end{array}\right) \mathcal{J}
$$

whenever the inverse above exists. In particular, we can see from Theorem 2.3 that $M_{k}(\lambda)$ is well defined for every $\lambda \in \mathbb{C} \backslash \mathbb{R}$ and $k=N+1$, when Hypothesis 2.1 holds. The following statement generalizes [1, Lemma 2.10$]$ to jointly varying endpoints.

Theorem 2.4 Let $\gamma \in \Omega, \lambda \in \mathbb{C}$, and $k \in[0, \infty)_{\mathbb{Z}}$. If $M_{k}(\lambda)$ and $M_{k}(\bar{\lambda})$ exist, then $M_{k}^{*}(\lambda)=$ $M_{k}(\bar{\lambda})$. Moreover, $M_{k}(\cdot)$ is an analytic function in its argument $\lambda$.

Proof This result follows from (4.11) and (4.9) via [1, Lemma 2.10]. 
For any $M \in \mathbb{C}^{2 n \times 2 n}$, we define the Weyl-solution $\mathcal{X}(\lambda)$ of $\left(\mathrm{S}_{\lambda}\right)$ with values in $\mathbb{C}^{4 n \times 2 n}$ by

$$
\mathcal{X}_{k}(\lambda):=\frac{1}{\sqrt{2}}\left(\begin{array}{cc}
\mathcal{J} & -\mathcal{J} \\
\Phi_{k}(\lambda) & \Phi_{k}(\lambda)
\end{array}\right)\left(\begin{array}{l}
\mathcal{J} \\
M
\end{array}\right)
$$

It then follows, compare with [1, Remark 2.12(i)], that $\gamma \mathcal{X}_{k}(\lambda)=0$ if and only if the matrix $M$ equals $M_{k}(\lambda)$ defined in (2.4).

One of the central concepts of this paper is the $\mathcal{E}(M)$ function with values in $\mathbb{C}^{2 n \times 2 n}$, through which we later on define the Weyl disks. For $M \in \mathbb{C}^{2 n \times 2 n}$, we put

$$
\mathcal{E}_{k}(M):=i \delta(\lambda) \mathcal{X}_{k}^{*}(\lambda)\left(\begin{array}{cc}
-\mathcal{J} & 0 \\
0 & \mathcal{J}
\end{array}\right) \mathcal{X}_{k}(\lambda)=\left(\begin{array}{c}
\mathcal{J} \\
M
\end{array}\right)^{*}\left(\begin{array}{cc}
\mathcal{H}_{k}(\lambda) & \mathcal{G}_{k}(\lambda) \\
\mathcal{G}_{k}(\lambda) & \mathcal{H}_{k}(\lambda)
\end{array}\right)\left(\begin{array}{l}
\mathcal{J} \\
M
\end{array}\right)
$$

where $\delta(\lambda):=\operatorname{sgnim}(\lambda)$ and

$$
\mathcal{H}_{k}(\lambda):=\frac{1}{2} i \delta(\lambda)\left[\Phi_{k}^{*}(\lambda) \mathcal{J} \Phi_{k}(\lambda)-\mathcal{J}\right], \quad \mathcal{G}_{k}(\lambda):=\mathcal{H}_{k}(\lambda)+i \delta(\lambda) \mathcal{J}
$$

From $(i \mathcal{J})^{*}=i \mathcal{J}$ one can see that $\mathcal{E}_{k}(M), \mathcal{H}_{k}(\lambda)$, and $\mathcal{G}_{k}(\lambda)$ are Hermitian matrices. Moreover, $\mathcal{H}_{0}(\lambda)=0$ and the Lagrange identity in [1, Theorem 2.6] implies the following crucial identities:

$$
\begin{aligned}
& \mathcal{E}_{k}(M)=-2 \delta(\lambda) \operatorname{im}(M)+|\operatorname{im}(\lambda)|\left(M^{*}-\mathcal{J}\right)\left[\sum_{j=0}^{k-1} \Phi_{j+1}^{*}(\lambda) \Psi_{j} \Phi_{j+1}(\lambda)\right](M+\mathcal{J}), \\
& \mathcal{H}_{k}(\lambda)=|\operatorname{im}(\lambda)| \sum_{j=0}^{k-1} \Phi_{j+1}^{*}(\lambda) \Psi_{j} \Phi_{j+1}(\lambda) .
\end{aligned}
$$

Since $\Phi(\lambda)$ is a fundamental matrix of $\left(S_{\lambda}\right)$, equality (2.8) then justifies the following result.

Theorem 2.5 If Hypothesis 2.2 holds, then the matrix $\mathcal{H}_{k}(\lambda)$ is positive definite for every $\lambda \in \mathbb{C} \backslash \mathbb{R}$ and $k \geq N_{0}+1$. In addition, for such $k$ we have (suppressing the argument $\lambda$ )

$$
\mathcal{E}_{k}(M)=-\mathcal{J}\left(\mathcal{H}_{k}-\mathcal{G}_{k} \mathcal{H}_{k}^{-1} \mathcal{G}_{k}\right) \mathcal{J}+\left(M^{*}-\mathcal{J} \mathcal{G}_{k} \mathcal{H}_{k}^{-1}\right) \mathcal{H}_{k}\left(M+\mathcal{H}_{k}^{-1} \mathcal{G}_{k} \mathcal{J}\right)
$$

Proof The invertibility of $\mathcal{H}_{k}(\lambda)$ for all $k \geq N_{0}+1$ follows from (2.8) and Hypothesis 2.2. Moreover, identity (2.9) is a consequence of (4.12) and (4.14).

For any $\lambda \in \mathbb{C} \backslash \mathbb{R}$, we now define the Weyl disk $D_{k}(\lambda)$ and the Weyl circle $C_{k}(\lambda)$ as

$$
D_{k}(\lambda):=\left\{M \in \mathbb{C}^{2 n \times 2 n}, \mathcal{E}_{k}(M) \leq 0\right\}, \quad C_{k}(\lambda):=\left\{M \in \mathbb{C}^{2 n \times 2 n}, \mathcal{E}_{k}(M)=0\right\}
$$

The following result provides some properties of the elements in $D_{k}(\lambda)$ and $C_{k}(\lambda)$. It is a generalization of [1, Theorems 3.2 and 3.3] to jointly varying endpoints.

Theorem 2.6 Let $\lambda \in \mathbb{C} \backslash \mathbb{R}, k \in[0, \infty)_{\mathbb{Z}}$, and $M \in \mathbb{C}^{2 n \times 2 n}$. Then the following hold.

(i) The matrix $M \in C_{k}(\lambda)$ if and only if there exists $\gamma \in \Omega$ such that $\gamma \mathcal{X}_{k}(\lambda)=0$. In this case, we have, with such a matrix $\gamma$, that $M=M_{k}(\lambda)$, whenever the matrix $M_{k}(\lambda)$ exists. 
(ii) The matrix $M$ satisfies $\mathcal{E}_{k}(M)<0$ if and only if there exists $\gamma \in \mathbb{C}^{2 n \times 4 n}$ such that $i \delta(\lambda) \gamma\left(\begin{array}{cc}-\mathcal{J} & 0 \\ 0 & \mathcal{J}\end{array}\right) \gamma^{*}>0$ and $\gamma \mathcal{X}_{k}(\lambda)=0$. In this case, we have, with such a matrix $\gamma$, that $M=M_{k}(\lambda)$, whenever the matrix $M_{k}(\lambda)$ exists, and $\gamma$ can be chosen so that $\gamma \gamma^{*}=I$.

(iii) We have $\mathcal{E}_{k}(-\mathcal{J})=-2 \delta(\lambda) i \mathcal{J}$, i.e., $-\mathcal{J} \notin D_{k}(\lambda)$.

Proof Statements (i) and (ii) follow by [1, Theorems 3.2-3.3] from the facts that $D_{k}(\lambda)$ and $C_{k}(\lambda)$ coincide respectively with the Weyl disk and Weyl circle in (4.13). Statement (iii) is verified by direct calculation from (2.6), since $i \mathcal{J}$ is indefinite.

The center $P_{k}(\lambda)$ and the matrix radius $R_{k}(\lambda)$ of the Weyl disk $D_{k}(\lambda)$ are defined as the $2 n \times 2 n$ matrices

$$
P_{k}(\lambda):=-\mathcal{H}_{k}^{-1}(\lambda) \mathcal{G}_{k}(\lambda) \mathcal{J}=-\mathcal{J}+i \delta(\lambda) \mathcal{H}_{k}^{-1}(\lambda), \quad R_{k}(\lambda):=\mathcal{H}_{k}^{-1 / 2}(\lambda)
$$

whenever $\mathcal{H}_{k}(\lambda)$ is invertible, i.e., whenever $\mathcal{H}_{k}(\lambda)>0$. The following theorem provides the most important geometric properties of the Weyl disks, including their nested property, closedness, and convexity. It is a generalization of [1, Theorems 3.6 and 3.8] to jointly varying endpoints. Let $\mathbb{V}$ and $\mathbb{U}$ be the sets of all complex contractive and unitary $2 n \times 2 n$ matrices, respectively, i.e., $\mathbb{V}:=\left\{V \in \mathbb{C}^{2 n \times 2 n}, V^{*} V \leq I\right\}$ and $\mathbb{U}:=\left\{U \in \mathbb{C}^{2 n \times 2 n}, U^{*} U=I\right\}$.

Theorem 2.7 Let $\lambda \in \mathbb{C} \backslash \mathbb{R}$. Then $D_{k}(\lambda) \subseteq D_{j}(\lambda)$ for every $k, j \in[0, \infty)_{\mathbb{Z}}$ with $k \geq j$. In addition, under Hypothesis 2.2 for every $k \geq N_{0}+1$, we have the representations

$$
\begin{aligned}
& D_{k}(\lambda)=\left\{P_{k}(\lambda)+R_{k}(\lambda) V R_{k}(\bar{\lambda}), V \in \mathbb{V}\right\}, \\
& C_{k}(\lambda)=\left\{P_{k}(\lambda)+R_{k}(\lambda) U R_{k}(\bar{\lambda}), U \in \mathbb{U}\right\} .
\end{aligned}
$$

Consequently, the Weyl disks $D_{k}(\lambda)$ are closed and convex for every $k \geq N_{0}+1$.

Proof The result follows from (4.15), (4.16), and (4.17) combined with Corollary 4.5.

The above theorem implies that the intersection of all the Weyl disks $D_{k}(\lambda)$ for $k \geq N_{0}+1$ is a nonempty, closed, and convex set. This yields that the limiting Weyl disk has the form

$$
D_{+}(\lambda):=\bigcap_{k \geq N_{0}+1} D_{k}(\lambda)=\left\{P_{+}(\lambda)+R_{+}(\lambda) V R_{+}(\bar{\lambda}), V \in \mathbb{V}\right\}
$$

where $P_{+}(\lambda)$ and $R_{+}(\lambda)$ are the $2 n \times 2 n$ matrices defined by

$$
P_{+}(\lambda):=\lim _{k \rightarrow \infty} P_{k}(\lambda), \quad R_{+}(\lambda):=\lim _{k \rightarrow \infty} R_{k}(\lambda) \geq 0
$$

They are called the center and the matrix radius of the limiting Weyl disk $D_{+}(\lambda)$. Note that the convergence of $P_{k}(\lambda)$ and $R_{k}(\lambda)$ can be seen from (2.10) and (2.8).

Remark 2.8 If we denote by $\mathcal{H}_{+}^{\text {inv }}(\lambda)$ the limit of $\mathcal{H}_{k}^{-1}(\lambda)$ as $k \rightarrow \infty$, which exists by (2.8), then the formulas in (2.11) for the center and matrix radius of the limiting Weyl disk reduce 


$$
P_{+}(\lambda)=-\mathcal{J}+i \delta(\lambda) \mathcal{H}_{+}^{\text {inv }}(\lambda), \quad R_{+}(\lambda)=\left[\mathcal{H}_{+}^{\text {inv }}(\lambda)\right]^{1 / 2}
$$

The next result is a generalization of [1, Corollary 3.12] to jointly varying endpoints. Note that as in Theorem 2.6(iii) we have $-\mathcal{J} \notin D_{+}(\lambda)$.

Theorem 2.9 Let $\lambda \in \mathbb{C} \backslash \mathbb{R}, M \in \mathbb{C}^{2 n \times 2 n}$, and suppose that Hypothesis 2.2 holds. Then $M$ belongs to the limiting Weyl disk $D_{+}(\lambda)$ if and only if

$$
\left(M^{*}-\mathcal{J}\right)\left[\sum_{k=0}^{\infty} \Phi_{k+1}^{*}(\lambda) \Psi_{k} \Phi_{k+1}(\lambda)\right](M+\mathcal{J}) \leq \frac{2 \operatorname{im}(M)}{\operatorname{im}(\lambda)} .
$$

Proof This statement follows from (2.7), or alternatively by [1, Corollary 3.12] from (4.18) and the definition of the Weyl solution in (4.11) and (4.8).

Remark 2.10 The limiting Weyl circle $C_{+}(\lambda)$ can be introduced as the boundary of the limiting Weyl disk $D_{+}(\lambda)$. Then $M \in C_{+}(\lambda)$ if and only if any of the following two equivalent conditions hold, compare with [1, Remark 3.18],

$$
\begin{aligned}
& \left(M^{*}-\mathcal{J}\right)\left[\sum_{k=0}^{\infty} \Phi_{k+1}^{*}(\lambda) \Psi_{k} \Phi_{k+1}(\lambda)\right](M+\mathcal{J})=\frac{2 \operatorname{im}(M)}{\operatorname{im}(\lambda)} \\
& \lim _{k \rightarrow \infty} \mathcal{X}_{k}^{*}(\lambda)\left(\begin{array}{cc}
-\mathcal{J} & 0 \\
0 & \mathcal{J}
\end{array}\right) \mathcal{X}_{k}(\lambda)=0
\end{aligned}
$$

We now discuss some properties of square summable solutions of system $\left(\mathrm{S}_{\lambda}\right)$. We say that a sequence $z=\left\{z_{k}\right\}_{k=0}^{\infty}$ with $z_{k} \in \mathbb{C}^{2 n}$ belongs to the space $\ell_{\Psi}^{2}$, i.e., $z$ is square summable, if

$$
\|z\|_{\Psi}<\infty, \quad \text { where }\|z\|_{\Psi}:=\left(\sum_{k=0}^{\infty} z_{k+1}^{*} \Psi_{k} z_{k+1}\right)^{1 / 2}=\lim _{N \rightarrow \infty} \sqrt{\langle z, z\rangle_{\Psi, N}} .
$$

For every $\lambda \in \mathbb{C} \backslash \mathbb{R}$, we denote the space of all square summable solutions of $\left(\mathrm{S}_{\lambda}\right)$ as

$$
\mathcal{N}(\lambda):=\left\{z \in \ell_{\Psi}^{2}[0, \infty)_{\mathbb{Z}}, z \text { solves }\left(\mathrm{S}_{\lambda}\right)\right\}
$$

From [1, Theorem 4.2] we know that $\operatorname{dim} \mathcal{N}(\lambda) \geq n$. According to the standard terminology, we say that system $\left(S_{\lambda}\right)$ is in the limit point case when $\operatorname{dim} \mathcal{N}(\lambda)=n$, and that system $\left(S_{\lambda}\right)$ is in the limit circle case when $\operatorname{dim} \mathcal{N}(\lambda)=2 n$. The following result is quite surprising in the sense that one would expect to have $R_{+}(\lambda)=0$ in the limit point case; see [1, Theorem 4.4]. To the contrary, due to the augmented structure of the matrix $R_{+}(\lambda)$, which has dimension $2 n$, it is the rank of $R_{+}(\lambda)$ alone which determines the number of linearly independent square summable solutions of $\left(\mathrm{S}_{\lambda}\right)$. In the result below, we show that $\operatorname{rank} R_{+}(\lambda) \geq n$, so that the equality $\operatorname{rank} R_{+}(\lambda)=n$ must necessarily hold in the limit point case. This fact is stated in Corollary 2.12 below and also illustrated in Example 3.5. 
Theorem 2.11 Let $\lambda \in \mathbb{C} \backslash \mathbb{R}$ and suppose that Hypothesis 2.2 holds. Then system $\left(\mathrm{S}_{\lambda}\right)$ has exactly $\operatorname{rank} R_{+}(\lambda)$ linearly independent square summable solutions, i.e.,

$$
n \leq \operatorname{dim} \mathcal{N}(\lambda)=\operatorname{rank} R_{+}(\lambda) \leq 2 n
$$

Proof The statement in (2.14) is proven in Theorem 4.6 and (4.19).

The meaning of Theorem 2.11 can be explained also directly from (2.8) and Remark 2.8. In particular, by (2.12), the rank of $R_{+}(\lambda)$ is equal to the number of positive eigenvalues of the matrix $\mathcal{H}_{+}^{\text {inv }}(\lambda)$ from Remark 2.8. This number is then the same as the number of the eigenvalues of $\mathcal{H}_{k}(\lambda)$, which tend to a finite limit as $k \rightarrow \infty$. Equation (2.8) then shows that this number is equal to the number of linearly independent square summable solutions of $\left(\mathrm{S}_{\lambda}\right)$.

Corollary 2.12 Let $\lambda \in \mathbb{C} \backslash \mathbb{R}$ and suppose that Hypothesis 2.2 holds. Then system $\left(\mathrm{S}_{\lambda}\right)$ is in the limit point case if and only if rank $R_{+}(\lambda)=n$, while $\left(\mathrm{S}_{\lambda}\right)$ is in the limit circle case if and only if $\operatorname{rank} R_{+}(\lambda)=2 n$.

Remark 2.13 When system $\left(\mathrm{S}_{\lambda}\right)$ has the special structure shown in (1.2), it can be deduced from [26, Corollary 4.6] that the total number of eigenvalues of (2.1) is equal to the dimension of the space of admissible functions for the associated discrete quadratic functional. In some even more special cases, such as for the second-order Sturm-Liouville difference equations with periodic or antiperiodic endpoints, this exact number of the eigenvalues of (2.1) is derived in [17, Theorem 4.2] or [27, Theorem 4.1]. The result in [26, Corollary 4.6] is based on the Rayleigh principle for system $\left(S_{\lambda}\right)$ with (1.5), compare also with [15, Theorem 3.2], and on the fact that the space of admissible functions is independent of $\lambda$ (as a consequence of the structure in (1.2)). As the Rayleigh principle for eigenvalue problems (2.1) is not known and the space of admissible functions is in this case not constant in $\lambda$, the question about the total number of eigenvalues of (2.1) remains open for the general linear dependence on $\lambda$. On the other hand, the oscillation theorem for discrete symplectic eigenvalue problems with jointly varying endpoints in [28, Theorem 6.13] yields that the total number of the eigenvalues of $(2.1)$ is less or equal to $(N+3) n$.

\section{Examples}

In this section we examine several examples which illustrate the presented theory. In particular, we consider the periodic and antiperiodic boundary conditions as in [15, Remark 6.17] and the corresponding $M(\lambda)$-function. To our knowledge, this form is now derived for the first time.

Example 3.1 For the periodic endpoints $z_{0}=z_{N+1}$, we take $\gamma=(1 / \sqrt{2})(\mathcal{J},-\mathcal{J}) \in \Omega$. In this case, the matrix in $(2.3)$ is $L(\lambda)=(1 / \sqrt{2}) \mathcal{J}\left[\Phi_{N+1}(\lambda)+\mathcal{J}\right]$. Then, by Theorem $2.3, \lambda \in \mathbb{C}$ is an eigenvalue of (2.1) if and only if the matrix $\Phi_{N+1}(\lambda)+\mathcal{J}$ is singular, and the number $\operatorname{dim} \operatorname{Ker}\left[\Phi_{N+1}(\lambda)+\mathcal{J}\right]$ is its multiplicity. Moreover, the $M(\lambda)$-function in (2.4) reduces to

$$
M_{k}(\lambda)=-\left[\Phi_{k}(\lambda)+\mathcal{J}\right]^{-1}\left[\Phi_{k}(\lambda)-\mathcal{J}\right] \mathcal{J}
$$


Example 3.2 For the antiperiodic endpoints $z_{0}+z_{N+1}=0$, we take $\gamma=(1 / \sqrt{2})(\mathcal{J}, \mathcal{J}) \in \Omega$. In this case we have $L(\lambda)=(1 / \sqrt{2}) \mathcal{J}\left[\Phi_{N+1}(\lambda)-\mathcal{J}\right]$ and, by Theorem $2.3, \lambda \in \mathbb{C}$ is an eigenvalue of (2.1) if and only if the matrix $\Phi_{N+1}(\lambda)-\mathcal{J}$ is singular. The multiplicity of $\lambda$ is then $\operatorname{dim} \operatorname{Ker}\left[\Phi_{N+1}(\lambda)-\mathcal{J}\right]$. In addition, the $M(\lambda)$-function in (2.4) now has the form

$$
M_{k}(\lambda)=-\left[\Phi_{k}(\lambda)-\mathcal{J}\right]^{-1}\left[\Phi_{k}(\lambda)+\mathcal{J}\right] \mathcal{J}
$$

We illustrate our new results on the scalar symplectic system

$$
z_{k+1}(\lambda)=\left(\begin{array}{cc}
1 & 1 \\
-\lambda & 1-\lambda
\end{array}\right) z_{k}(\lambda), \quad \Psi_{k} \equiv \Psi:=\left(\begin{array}{ll}
1 & 0 \\
0 & 0
\end{array}\right) \text {. }
$$

This system corresponds to the second-order Sturm-Liouville difference equation

$$
-\Delta\left(p_{k} \Delta x_{k}\right)+q_{k} x_{k+1}=\lambda w_{k} x_{k+1}, \quad \text { where } p_{k}=w_{k} \equiv 1, q_{k} \equiv 0,
$$

compare with (1.2), which was extensively studied, e.g., in [17, 20, 29-34] and [16, Chapter 7]. System (3.1) satisfies the strong Atkinson condition in Hypothesis 2.1 or 2.2 with $N_{0}=1$, as can be easily verified.

Example 3.3 Consider the scalar eigenvalue problem with periodic endpoints

$$
\text { (3.1), } \quad k \in[0,3]_{\mathbb{Z}}, \quad z_{0}=z_{4},
$$

i.e., we look for the solutions of (3.1) with period 4. This problem corresponds to the periodic Sturm-Liouville eigenvalue problem (3.2) with $k \in[0,3]_{\mathbb{Z}}$ and $x_{0}=x_{4}, \Delta x_{0}=\Delta x_{4}$, which is studied in [16, Example 7.6]. It is shown in this reference that $\lambda=2$ is a double eigenvalue of (3.3) by finding two linearly independent eigenfunctions. The results in Theorem 2.3 and Example 3.1 confirm this conclusion. The fundamental matrix in (2.2) now satisfies

$$
\begin{aligned}
& \Phi_{2}(\lambda)=\left(\begin{array}{cc}
-\lambda+2 & \lambda-1 \\
\lambda^{2}-3 \lambda+1 & -\lambda^{2}+2 \lambda
\end{array}\right), \\
& \Phi_{4}(\lambda)=\left(\begin{array}{cc}
-\lambda^{3}+6 \lambda^{2}-10 \lambda+4 & \lambda^{3}-5 \lambda^{2}+6 \lambda-1 \\
\lambda^{4}-7 \lambda^{3}+15 \lambda^{2}-10 \lambda+1 & -\lambda^{4}+6 \lambda^{3}-10 \lambda^{2}+4 \lambda
\end{array}\right) .
\end{aligned}
$$

This yields that $\operatorname{det}\left[\Phi_{4}(\lambda)+\mathcal{J}\right]=-\lambda(\lambda-4)(\lambda-2)^{2}$. Thus, by Theorem 2.3 and Example 3.1, $\lambda=2$ is indeed a double eigenvalue of (3.3), and the columns of $\Phi(2)$ are the two linearly independent eigenfunctions. Note that $\Phi_{4}(2)=-\mathcal{J}=\Phi_{0}(2)$. The other eigenvalues of (3.3) are $\lambda=0$ with the eigenfunction $\Phi(0)(0,1)^{*}$, and $\lambda=4$ with the eigenfunction $\Phi(4)(2,1)^{*}$.

Example 3.4 Consider system (3.1), but now only on the interval $[0,2]_{\mathbb{Z}}$ and with the antiperiodic boundary conditions $z_{0}=-z_{2}$. From (3.4) we see that $\operatorname{det}\left[\Phi_{2}(\lambda)-\mathcal{J}\right]=(\lambda-2)^{2}$. Hence, by Theorem 2.3 and Example 3.2, $\lambda=2$ is a double eigenvalue of this problem with the columns of $\Phi(2)$ as the two linearly independent eigenfunctions. Note that $\Phi_{2}(2)=\mathcal{J}=-\Phi_{0}(2)$. This problem then does not have any other eigenvalues. 
In the last example, we calculate the rank of the limiting matrix radius $R_{+}(\lambda)$ and compare it with the corresponding number of linearly independent square summable solutions.

Example 3.5 We examine system $(3.1)$ on $[0, \infty)_{\mathbb{Z}}$ with the particular choice of $\lambda_{0} \in$ $\mathbb{C} \backslash \mathbb{R}$. We will show that system (3.1) with $\lambda=\lambda_{0}$ is in the limit point case, so that by $\left[1\right.$, Corollary 4.19] it is in the limit point case for every $\lambda \in \mathbb{C} \backslash \mathbb{R}$. Let $\lambda_{0}=2+i 2 \sqrt{3}$, i.e., system (3.1) reduces to the second-order difference equation $x_{k+2}+i 2 \sqrt{3} x_{k+1}+x_{k}=0$ on $[0, \infty)_{\mathbb{Z}}$. The roots of the corresponding characteristic polynomial are $v_{ \pm}:=( \pm 2-\sqrt{3}) i$, so that the fundamental matrix of (3.1) satisfying (2.2) has the form

$$
\Phi_{k}\left(\lambda_{0}\right)=\left(\begin{array}{cc}
v_{+}^{k} & v_{-}^{k} \\
v_{+}^{k}\left(v_{+}-1\right) & v_{-}^{k}\left(v_{-}-1\right)
\end{array}\right) P, \quad P:=\frac{1}{4}\left(\begin{array}{cc}
-i & -2-\sqrt{3}+i \\
i & -2+\sqrt{3}-i
\end{array}\right) .
$$

By (2.8), we obtain with $\mu_{ \pm}:=\left|\nu_{ \pm}\right|^{2}=7 \mp 4 \sqrt{3}$ that

$$
\mathcal{H}_{k}\left(\lambda_{0}\right)=2 \sqrt{3} P^{*} \sum_{j=0}^{k-1}\left(\begin{array}{cc}
\left|v_{+}\right|^{2 j+2} & \left(\bar{v}_{+} \nu_{-}\right)^{j+1} \\
\left(v_{+} \bar{v}_{-}\right)^{j+1} & \left|v_{-}\right|^{2 j+2}
\end{array}\right) P=2 \sqrt{3} P^{*} \sum_{j=0}^{k-1}\left(\begin{array}{ll}
\left(\mu_{+}\right)^{j+1} & (-1)^{j+1} \\
(-1)^{j+1} & \left(\mu_{-}\right)^{j+1}
\end{array}\right) P .
$$

Since each entry of $\mathcal{H}_{k}\left(\lambda_{0}\right)$ represents a geometric series, it can be evaluated explicitly as

$$
\mathcal{H}_{k}\left(\lambda_{0}\right)=2 \sqrt{3} P^{*}\left(\begin{array}{cc}
\left(\mu_{+}\right)\left[1-\left(\mu_{+}\right)^{k}\right] /\left(1-\mu_{+}\right) & {\left[(-1)^{k}-1\right] / 2} \\
{\left[(-1)^{k}-1\right] / 2} & \left(\mu_{-}\right)\left[1-\left(\mu_{-}\right)^{k}\right] /\left(1-\mu_{-}\right)
\end{array}\right) P .
$$

Therefore, the matrix $\mathcal{H}_{k}\left(\lambda_{0}\right)$ is indeed invertible (positive definite) for all $k \geq N_{0}+1=2$ and, by Remark 2.8 , we have

$$
\begin{aligned}
& \mathcal{H}_{+}^{\mathrm{inv}}\left(\lambda_{0}\right)=\lim _{k \rightarrow \infty} \mathcal{H}_{k}^{-1}\left(\lambda_{0}\right)=\left(\begin{array}{cc}
4 & 2+\sqrt{3}-i \\
2+\sqrt{3}+i & 2+\sqrt{3}
\end{array}\right), \\
& P_{+}\left(\lambda_{0}\right)=-\mathcal{J}+i \mathcal{H}_{+}^{\mathrm{inv}}\left(\lambda_{0}\right)=\left(\begin{array}{cc}
4 i & (2+\sqrt{3}) i \\
(2+\sqrt{3}) i & (2+\sqrt{3}) i
\end{array}\right) .
\end{aligned}
$$

The matrix $R_{+}\left(\lambda_{0}\right)$ can also be calculated explicitly by (2.12), but it is not really important. We can find the eigenvalues of $R_{+}\left(\lambda_{0}\right)$ as the nonnegative square roots of the eigenvalues of $\mathcal{H}_{+}^{\text {inv }}\left(\lambda_{0}\right)$. Namely, since the eigenvalues of $\mathcal{H}_{+}^{\text {inv }}\left(\lambda_{0}\right)$ are 0 and $6+\sqrt{3}$, we get rank $R_{+}\left(\lambda_{0}\right)=1$ and system (3.1) is in the limit point case, by Corollary 2.12. The square summable solution of (3.1) is then given as the second component of the columns of the Weyl solution $\mathcal{X}_{+}\left(\lambda_{0}\right)$ in (2.5) with $M=P_{+}\left(\lambda_{0}\right)$. That is, the columns of the matrix $\Phi\left(\lambda_{0}\right) \mathcal{H}_{+}^{\text {inv }}\left(\lambda_{0}\right)$ are square summable. But since $\mathcal{H}_{+}^{\text {inv }}\left(\lambda_{0}\right)$ is singular, it follows that the square summable solutions of (3.1) are generated by exactly one column of the matrix $\Phi\left(\lambda_{0}\right) \mathcal{H}_{+}^{\text {inv }}\left(\lambda_{0}\right)$. On the other hand, since $\left|v_{+}\right|<1$, one can identify the first column of $\Phi\left(\lambda_{0}\right) P^{-1}$ in (3.6) as the square summable solution of (3.1).

\section{Augmented symplectic system}

We shall show that problem (2.1) is equivalent with a certain eigenvalue problem in dimension $4 n$ with separated endpoints. Define the $4 n \times 4 n$ matrices (the augmentation is 
emphasized by the bold notation)

$$
\begin{aligned}
& \mathcal{S}_{k}:=\left(\begin{array}{cc}
I & 0 \\
0 & \mathcal{S}_{k}
\end{array}\right), \quad \mathcal{V}_{k}:=\left(\begin{array}{cc}
0 & 0 \\
0 & \mathcal{V}_{k}
\end{array}\right), \quad \Psi_{k}:=\left(\begin{array}{cc}
0 & 0 \\
0 & \Psi_{k}
\end{array}\right) \geq 0, \\
& \boldsymbol{\Phi}_{k}(\lambda):=\frac{1}{\sqrt{2}}\left(\begin{array}{cc}
\mathcal{J} & -\mathcal{J} \\
\Phi_{k}(\lambda) & \Phi_{k}(\lambda)
\end{array}\right), \quad \mathcal{J}:=\left(\begin{array}{cc}
-\mathcal{J} & 0 \\
0 & \mathcal{J}
\end{array}\right), \quad \mathcal{K}:=\left(\begin{array}{cc}
0 & \mathcal{J} \\
\mathcal{J} & 0
\end{array}\right) .
\end{aligned}
$$

Then one can easily verify that $\mathcal{J}^{*}=-\mathcal{J}=\mathcal{J}^{-1}, \mathcal{K}^{*}=-\mathcal{K}=\mathcal{K}^{-1}$, and

$$
\mathcal{S}_{k}^{*} \mathcal{J} \mathcal{S}_{k}=\mathcal{J}, \quad \mathcal{S}_{k}^{*} \mathcal{J} \mathcal{V}_{k} \quad \text { is Hermitian, } \quad \mathcal{V}_{k}^{*} \mathcal{J} \mathcal{V}_{k}=0, \quad \boldsymbol{\Psi}_{k}=\mathcal{J} \mathcal{V}_{k} \mathcal{J} \mathcal{S}_{k}^{*} \mathcal{J}
$$

Consider the augmented symplectic system

$$
\boldsymbol{z}_{k+1}(\lambda)=\left(\mathcal{S}_{k}+\lambda \mathcal{V}_{k}\right) \boldsymbol{z}_{k}(\lambda)
$$

It follows that $\boldsymbol{\Phi}(\lambda)$ is a fundamental matrix of system $\left(\mathbf{S}_{\lambda}\right)$, because

$$
\boldsymbol{\Phi}_{k}(\lambda)=\left(\begin{array}{cc}
I & 0 \\
0 & \Phi_{k}(\lambda) \mathcal{J}
\end{array}\right) \boldsymbol{Q} \text { with } \boldsymbol{Q}:=\boldsymbol{\Phi}_{0}(\lambda)=\frac{1}{\sqrt{2}}\left(\begin{array}{cc}
\mathcal{J} & -\mathcal{J} \\
-\mathcal{J} & -\mathcal{J}
\end{array}\right), \operatorname{det} \boldsymbol{Q}=1
$$

where $\Phi_{k}(\lambda) \mathcal{J}$ is the fundamental matrix of the original system $\left(\mathrm{S}_{\lambda}\right)$ starting with the initial value $\Phi_{0}(\lambda) \mathcal{J}=I$. Vector solutions $\boldsymbol{z}(\lambda)$ of $\left(\mathbf{S}_{\lambda}\right)$ are in dimension $4 n$ and the matrix solutions $\boldsymbol{Z}(\lambda)$ of $\left(\mathbf{S}_{\lambda}\right)$ are in dimension $4 n \times 2 n$. It follows that they have the form

$$
\mathbf{z}_{k}(\lambda)=\left(\begin{array}{c}
d \\
z_{k}(\lambda)
\end{array}\right), \quad \boldsymbol{z}_{k}(\lambda)=\left(\begin{array}{cc}
D & E \\
Z_{k}(\lambda) & \widetilde{Z}_{k}(\lambda)
\end{array}\right)
$$

where $d \in \mathbb{C}^{n}$ is a constant vector, $D, E \in \mathbb{C}^{2 n \times n}$ are constant matrices, $z(\lambda)$ is a vector solution of $\left(\mathrm{S}_{\lambda}\right)$ in dimension $2 n$, and $Z(\lambda), \widetilde{Z}(\lambda)$ are matrix solutions of $\left(\mathrm{S}_{\lambda}\right)$ in dimension $2 n \times n$. It turns out that the main properties of discrete symplectic systems $\left(\mathrm{S}_{\lambda}\right)$ and their solutions, such as those in [1, Section 2] or [5, Section 2], are preserved for the augmented system $\left(\mathbf{S}_{\lambda}\right)$. In particular, we have the following identities for the coefficients of $\left(\mathbf{S}_{\lambda}\right)$ :

$$
\left(\mathcal{S}_{k}+\lambda \mathcal{V}_{k}\right)^{*} \mathcal{J}\left(\mathcal{S}_{k}+\bar{\lambda} \mathcal{V}_{k}\right)=\mathcal{J}, \quad\left(\mathcal{S}_{k}+\lambda \mathcal{V}_{k}\right)^{-1}=-\mathcal{J}\left(\mathcal{S}_{k}^{*}+\lambda \mathcal{V}_{k}^{*}\right) \mathcal{J}
$$

and the Lagrange identity for two $4 n \times m$ matrix solutions of $\left(\mathbf{S}_{\lambda}\right)$ and $\left(\mathbf{S}_{v}\right)$

$$
\boldsymbol{Z}_{k+1}^{*}(\lambda) \mathcal{J} \boldsymbol{Z}_{k+1}(v)=\boldsymbol{Z}_{0}^{*}(\lambda) \mathcal{J} \boldsymbol{Z}_{0}(v)+(\bar{\lambda}-v) \sum_{j=0}^{k} \boldsymbol{Z}_{j+1}^{*}(\lambda) \boldsymbol{\Psi}_{j} \mathbf{Z}_{j+1}(v)
$$

for all $k \in[0, \infty)_{\mathbb{Z}}$. In addition, the fundamental matrix $\boldsymbol{\Phi}(\lambda)$ of $\left(\mathbf{S}_{\lambda}\right)$ satisfies

$$
\boldsymbol{\Phi}_{k}^{*}(\lambda) \mathcal{J} \boldsymbol{\Phi}_{k}(\bar{\lambda})=\mathcal{K}, \quad \boldsymbol{\Phi}_{k}(\bar{\lambda}) \mathcal{K} \boldsymbol{\Phi}_{k}^{*}(\lambda)=\mathcal{J}, \quad \boldsymbol{\Phi}_{0}^{*}(\lambda) \boldsymbol{\Phi}_{0}(\lambda)=I,
$$

i.e., the matrix $\boldsymbol{\Phi}_{0}(\lambda)$ is unitary. 
With the above preliminary setting, we now consider the augmented eigenvalue problem

$$
\left(\mathbf{S}_{\lambda}\right), \quad k \in[0, N]_{\mathbb{Z}}, \lambda \in \mathbb{C}, \quad \boldsymbol{\alpha} \boldsymbol{z}_{0}=0, \quad \boldsymbol{\beta} \boldsymbol{z}_{N+1}=0,
$$

where the $2 n \times 4 n$ matrices $\boldsymbol{\alpha}, \boldsymbol{\beta} \in \boldsymbol{\Gamma}:=\Omega$ are defined by

$$
\boldsymbol{\alpha}:=\frac{1}{\sqrt{2}}(-I \quad I), \quad \boldsymbol{\beta}:=\gamma .
$$

Equation (4.3) and the above choice of $\boldsymbol{\alpha}$ imply that the solutions of (4.5) have the form

$$
\boldsymbol{z}_{k}(\lambda)=\left(\begin{array}{l}
z_{0}(\lambda) \\
z_{k}(\lambda)
\end{array}\right), \quad \text { where } z(\lambda) \text { is a solution of }(2.1) .
$$

Conversely, every solution $z(\lambda)$ of (2.1) defines through (4.7) a solution $\boldsymbol{z}(\lambda)$ of (4.5). In addition, the form of the matrix $\boldsymbol{\Psi}_{k}$ in (4.1) implies that the semi-norm of the augmented solution $\mathbf{z}(\lambda)$ is the same as the semi-norm of the generating solution $z(\lambda)$, because

$$
\begin{aligned}
& \langle\mathbf{z}, \tilde{\mathbf{z}}\rangle_{\Psi, N}:=\sum_{k=0}^{N} \mathbf{z}_{k+1}^{*} \boldsymbol{\Psi}_{k} \mathbf{z}_{k+1}=\sum_{k=0}^{N} z_{k+1}^{*} \Psi_{k} \tilde{z}_{k+1}=\langle z, \tilde{z}\rangle_{\Psi, N}, \\
& \boldsymbol{z}_{k}=\left(\begin{array}{c}
z_{0} \\
z_{k}
\end{array}\right), \quad \tilde{\mathbf{z}}_{k}=\left(\begin{array}{c}
\tilde{z}_{0} \\
\tilde{z}_{k}
\end{array}\right) .
\end{aligned}
$$

Following the theory in [1, Sections 2-3], we consider the fundamental matrix $\widetilde{\boldsymbol{\Phi}}(\lambda)$ of the augmented system $\left(\mathbf{S}_{\lambda}\right)$ satisfying the initial condition $\widetilde{\boldsymbol{\Phi}}_{0}(\lambda)=\left(\boldsymbol{\alpha}^{*},-\mathcal{J} \boldsymbol{\alpha}^{*}\right)$. This means that

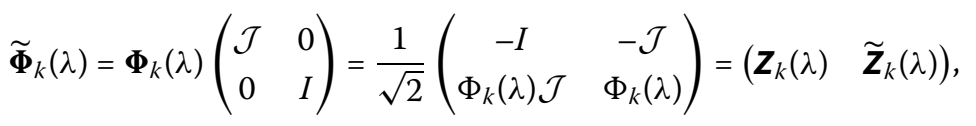

where $\boldsymbol{Z}(\lambda)$ and $\tilde{\boldsymbol{Z}}(\lambda)$ are normalized conjoined bases of $\left(\mathbf{S}_{\lambda}\right)$ defined by

$$
\boldsymbol{Z}_{k}(\lambda):=\frac{1}{\sqrt{2}}\left(\begin{array}{c}
-I \\
\Phi_{k}(\lambda) \mathcal{J}
\end{array}\right), \quad \widetilde{\boldsymbol{Z}}_{k}(\lambda):=\frac{1}{\sqrt{2}}\left(\begin{array}{c}
-\mathcal{J} \\
\Phi_{k}(\lambda)
\end{array}\right) .
$$

The solution $\tilde{\boldsymbol{Z}}(\lambda)$ is called the natural conjoined basis of $\left(\mathbf{S}_{\lambda}\right)$. We have already mentioned in Section 1 that only the corresponding weak Atkinson condition is required when studying the spectral problem with separated endpoints. Applying this requirement to the augmented problem (4.5), we can see that we need to assume

$$
\sum_{k=0}^{N_{0}} \boldsymbol{z}_{k+1}^{*}(\lambda) \boldsymbol{\Psi}_{k} \boldsymbol{z}_{k+1}(\lambda)>0
$$

for certain solutions $\boldsymbol{z}(\lambda)$ of $\left(\mathbf{S}_{\lambda}\right)$. This is formulated in the next assumptions.

Hypothesis 4.1 (Weak augmented Atkinson condition - finite) Inequality (4.10) is satisfied with $N_{0}=N$ for every column $\boldsymbol{z}(\lambda)$ of the natural conjoined basis $\widetilde{\boldsymbol{Z}}(\lambda)$ of $\left(\mathbf{S}_{\lambda}\right)$ on $[0, N+1]_{\mathbb{Z}}$ and every $\lambda \in \mathbb{C}$. 
Hypothesis 4.2 (Weak augmented Atkinson condition - infinite) There exists $N_{0} \in \mathbb{N}$ such that inequality (4.10) holds for every column $\boldsymbol{z}(\lambda)$ of the natural conjoined basis $\tilde{\boldsymbol{Z}}(\lambda)$ of $\left(\mathbf{S}_{\lambda}\right)$ on $[0, \infty)_{\mathbb{Z}}$ and every $\lambda \in \mathbb{C}$.

The next theorem provides basic properties of the eigenvalue problem (4.5).

Theorem 4.3 Let $\boldsymbol{\alpha}, \boldsymbol{\beta} \in \boldsymbol{\Gamma}$ be arbitrary and $N \in \mathbb{N}$. Then the following statements hold.

(i) Under Hypothesis 4.1, the eigenvalues of (4.5) are real and the eigenfunctions corresponding to different eigenvalues are orthogonal with respect to the semi-inner product $\langle\cdot, \cdot\rangle_{\boldsymbol{\Psi}, N}$.

(ii) A number $\lambda \in \mathbb{C}$ is an eigenvalue of (4.5) if and only if the matrix $\mathbf{L}(\lambda):=\boldsymbol{\beta} \widetilde{\boldsymbol{Z}}_{N+1}(\lambda)$ is singular. In this case, the eigenfunctions of (4.5) corresponding to the eigenvalue $\lambda$ have the form $z=\widetilde{\boldsymbol{Z}}_{k}(\lambda) d$ on $[0, N+1]_{\mathbb{Z}}$ with a nonzero $d \in \operatorname{Ker} \boldsymbol{L}(\lambda)$. Moreover, the geometric and algebraic multiplicities of $\lambda_{0}$ coincide and are equal to $\operatorname{dim} \operatorname{Ker} L(\lambda)$.

Proof The statement follows from [1, Theorem 2.8], when it is applied to the augmented eigenvalue problem (4.5).

When we write the weak Atkinson condition in (4.10) in terms of the data of the original problem (2.1), we get by (4.1) and (4.9) that

$$
\sum_{k=0}^{N_{0}} \widetilde{\mathbf{Z}}_{k+1}^{*}(\lambda) \Psi_{k} \widetilde{\mathbf{Z}}_{k+1}(\lambda)=\sum_{k=0}^{N_{0}} \Phi_{k+1}^{*}(\lambda) \Psi_{k} \Phi_{k+1}(\lambda)>0 .
$$

This shows that the two conditions in Hypotheses 4.1 and 2.1 are intimately connected, as stated in the following corollaries.

Corollary 4.4 System $\left(\mathrm{S}_{\lambda}\right)$ satisfies the strong Atkinson condition on $[0, N+1]_{\mathbb{Z}}$ (Hypothesis 2.1) if and only if the augmented system $\left(\mathbf{S}_{\lambda}\right)$ satisfies the corresponding weak Atkinson condition on $[0, N+1]_{\mathbb{Z}}$ (Hypothesis 4.1 ).

Corollary 4.5 System $\left(\mathrm{S}_{\lambda}\right)$ satisfies the strong Atkinson condition on $[0, \infty)_{\mathbb{Z}}$ (Hypothesis 2.2) if and only if the augmented system $\left(\mathbf{S}_{\lambda}\right)$ satisfies the corresponding weak Atkinson condition on $[0, \infty)_{\mathbb{Z}}$ (Hypothesis 4.2$)$.

In particular, we can see why assuming the weak Atkinson condition in the spectral theory for separated endpoints is really essential - the transformation of problem (2.1) with jointly varying endpoints, which satisfies the strong Atkinson condition, leads to the augmented problem (4.5) satisfying the corresponding weak Atkinson condition. Therefore, one can simply apply the previous results on separated endpoints to the augmented problem and then transform the obtained results back to the data of the original problem (2.1).

Following [1, Definitions 2.9 and 2.11], we define the $\boldsymbol{M}(\lambda)$-function for the augmented problem (4.5) and for $M \in \mathbb{C}^{2 n \times 2 n}$ the corresponding Weyl solution as

$$
\boldsymbol{M}_{k}(\lambda):=-\left[\boldsymbol{\beta} \widetilde{\boldsymbol{Z}}_{k}(\lambda)\right]^{-1} \boldsymbol{\beta} \boldsymbol{Z}_{k}(\lambda), \quad \boldsymbol{X}_{k}(\lambda):=\widetilde{\boldsymbol{\Phi}}_{k}(\lambda)\left(\begin{array}{c}
I \\
M
\end{array}\right)
$$


where $\boldsymbol{Z}(\lambda), \widetilde{\boldsymbol{Z}}(\lambda)$, and $\widetilde{\boldsymbol{\Phi}}(\lambda)$ are given in (4.9) and (4.8). In addition, for $M \in \mathbb{C}^{2 n \times 2 n}$, we define the $\mathcal{E}(M)$ function by

$$
\mathcal{E}_{k}(M):=i \delta(\lambda) \mathcal{X}_{k}^{*}(\lambda) \mathcal{J} \mathcal{X}_{k}(\lambda)=\left(\begin{array}{c}
I \\
M
\end{array}\right)^{*}\left(\begin{array}{cc}
\mathcal{F}_{k}(\lambda) & \mathcal{G}_{k}^{*}(\lambda) \\
\mathcal{G}_{k}(\lambda) & \mathcal{H}_{k}(\lambda)
\end{array}\right)\left(\begin{array}{c}
I \\
M
\end{array}\right)
$$

where $\mathcal{F}_{k}(\lambda), \mathcal{G}_{k}(\lambda)$, and $\mathcal{H}_{k}(\lambda)$ are $2 n \times 2 n$ matrices

$$
\begin{aligned}
& \mathcal{H}_{k}(\lambda):=i \delta(\lambda) \widetilde{\mathbf{Z}}_{k}^{*}(\lambda) \mathcal{J} \widetilde{\mathbf{Z}}_{k}(\lambda)=\frac{1}{2} i \delta(\lambda)\left[\Phi_{k}^{*}(\lambda) \mathcal{J} \Phi_{k}(\lambda)-\mathcal{J}\right] \\
& \mathcal{F}_{k}(\lambda):=i \delta(\lambda) \mathbf{Z}_{k}^{*}(\lambda) \mathcal{J} \boldsymbol{Z}_{k}(\lambda)=\mathcal{J}^{*} \mathcal{H}_{k}(\lambda) \mathcal{J}, \\
& \mathcal{G}_{k}(\lambda):=i \delta(\lambda) \widetilde{\mathbf{Z}}_{k}^{*}(\lambda) \mathcal{J} \boldsymbol{Z}_{k}(\lambda)=\mathcal{H}_{k}(\lambda) \mathcal{J}-i \delta(\lambda) .
\end{aligned}
$$

As in [1, Definition 3.1], the Weyl disk $\boldsymbol{D}_{k}(\lambda)$ and the Weyl circle $\boldsymbol{C}_{k}(\lambda)$ are defined by

$$
\boldsymbol{D}_{k}(\lambda):=\left\{M \in \mathbb{C}^{2 n \times 2 n}, \mathcal{E}_{k}(M) \leq 0\right\}, \quad \boldsymbol{C}_{k}(\lambda):=\left\{M \in \mathbb{C}^{2 n \times 2 n}, \mathcal{E}_{k}(M)=0\right\} .
$$

Note that under Hypothesis 4.2 the matrices $\mathcal{H}_{k}(\lambda)$ are positive definite (and hence invertible) for all $k \geq N_{0}+1$, because by (4.4) we have

$$
\mathcal{H}_{k}(\lambda)=2|\operatorname{im}(\lambda)| \sum_{j=0}^{k-1} \widetilde{\boldsymbol{Z}}_{j+1}^{*}(\lambda) \boldsymbol{\Psi}_{j} \widetilde{\boldsymbol{Z}}_{j+1}(\lambda)
$$

By [1, Theorem 3.8], the Weyl disk and the Weyl circle possess the representations

$$
\begin{aligned}
& \boldsymbol{D}_{k}(\lambda)=\left\{\boldsymbol{P}_{k}(\lambda)+\boldsymbol{R}_{k}(\lambda) V \boldsymbol{R}_{k}(\bar{\lambda}), V \in \mathbb{V}\right\}, \\
& \boldsymbol{C}_{k}(\lambda)=\left\{\boldsymbol{P}_{k}(\lambda)+\boldsymbol{R}_{k}(\lambda) U \boldsymbol{R}_{k}(\bar{\lambda}), U \in \mathbb{U}\right\},
\end{aligned}
$$

where $\mathbb{V}$ and $\mathbb{U}$ are the sets of all complex contractive and unitary $2 n \times 2 n$ matrices, respectively (as in Section 2), and where the center $\boldsymbol{P}_{k}(\lambda)$ and the matrix radius $\boldsymbol{R}_{k}(\lambda)$ are defined by

$$
\boldsymbol{P}_{k}(\lambda):=-\mathcal{H}_{k}^{-1}(\lambda) \mathcal{G}_{k}(\lambda)=-\mathcal{J}+i \delta(\lambda) \mathcal{H}_{k}^{-1}(\lambda), \quad \boldsymbol{R}_{k}(\lambda):=\mathcal{H}_{k}^{-1 / 2}(\lambda)
$$

Therefore, the Weyl disks $\boldsymbol{D}_{k}(\lambda)$ are closed, convex, and nested, so that the limiting Weyl disk

$$
\boldsymbol{D}_{+}(\lambda):=\bigcap_{k \geq N_{0}+1} \boldsymbol{D}_{k}(\lambda)=\left\{\boldsymbol{P}_{+}(\lambda)+\boldsymbol{R}_{+}(\lambda) V \boldsymbol{R}_{+}(\bar{\lambda}), V \in \mathbb{V}\right\}
$$

exists and is nonempty, closed, and convex as well. By using [1, Theorem 3.9] and the monotonicity of $\mathcal{H}_{k}(\lambda)$ shown in (4.14), the center and the matrix radius of $\boldsymbol{D}_{+}(\lambda)$ are

$$
\boldsymbol{P}_{+}(\lambda):=\lim _{k \rightarrow \infty} \boldsymbol{P}_{k}(\lambda), \quad \boldsymbol{R}_{+}(\lambda):=\lim _{k \rightarrow \infty} \boldsymbol{R}_{k}(\lambda) \geq 0 .
$$


Let $\ell_{\Psi}^{2}$ be the space of all square summable sequences $\boldsymbol{z}=\left\{\boldsymbol{z}_{k}\right\}_{k=0}^{\infty}$ on $[0, \infty)_{\mathbb{Z}}$ such that $\boldsymbol{z}_{k} \in \mathbb{C}^{4 n}$ with the semi-norm

$$
\|\boldsymbol{z}\|_{\Psi}<\infty, \quad \text { where }\|\boldsymbol{z}\|_{\Psi}:=\left(\sum_{k=0}^{\infty} \boldsymbol{z}_{k+1}^{*} \boldsymbol{\Psi}_{k} \boldsymbol{z}_{k+1}\right)^{1 / 2}=\lim _{N \rightarrow \infty} \sqrt{\langle\boldsymbol{z}, \boldsymbol{z}\rangle_{\Psi, N}} .
$$

For every $\lambda \in \mathbb{C} \backslash \mathbb{R}$, we denote the space of all square summable solutions of $\left(\mathbf{S}_{\lambda}\right)$ as

$$
\mathcal{N}(\lambda):=\left\{\boldsymbol{z} \in \ell_{\Psi}^{2}[0, \infty)_{\mathbb{Z}}, \boldsymbol{z} \text { solves }\left(\mathbf{S}_{\lambda}\right)\right\}
$$

From [1, Theorem 4.2] we know that the dimension of $\mathcal{N}(\lambda)$ is at least $2 n$, or more precisely

$$
\operatorname{dim} \mathcal{N}(\lambda)=2 n+\operatorname{rank} \boldsymbol{R}_{+}(\lambda)
$$

by [1, Theorem 4.9]. On the other hand, the analysis of the structure of the $\ell_{\Psi}^{2}$ solutions of the augmented system $\left(\mathbf{S}_{\lambda}\right)$ yields the following result.

Theorem 4.6 Let $\lambda \in \mathbb{C} \backslash \mathbb{R}$ and suppose that Hypothesis 4.2 holds. Then

$$
\operatorname{dim} \mathcal{N}(\lambda)=2 n+\operatorname{dim} \mathcal{N}(\lambda) \geq 3 n \text { and } n \leq \operatorname{rank} \boldsymbol{R}_{+}(\lambda) \leq 2 n,
$$

where $\mathcal{N}(\lambda)$ is the space of all $\ell_{\Psi}^{2}$ solutions of the original system $\left(S_{\lambda}\right)$, as defined in (2.13).

Proof Let $e_{j} \in \mathbb{C}^{2 n}$ be the $j$ th canonical unit vector for $j \in\{1, \ldots, 2 n\}$. Then the constant solutions $\boldsymbol{z}^{[j]}:=\left(e_{j}^{*}, 0^{*}\right)^{*} \in \mathbb{C}^{4 n}, j \in\{1, \ldots, 2 n\}$, certainly belong to $\mathcal{N}(\lambda)$, because $\left\|\boldsymbol{z}^{[j]}\right\|_{\boldsymbol{\Psi}}=0$. In addition, any square summable solution $z \in \mathcal{N}(\lambda)$ naturally generates a square summable solution $\mathbf{z}=\left(0^{*}, z^{*}\right)^{*} \in \mathcal{N}(\lambda)$, which is linearly independent with the above defined solutions $\boldsymbol{z}^{[1]}, \ldots, \boldsymbol{z}^{[2 n]}$. This yields that $\operatorname{dim} \mathcal{N}(\lambda)=2 n+\operatorname{dim} \mathcal{N}(\lambda)$. Moreover, since by [1, Theorem 4.2] we have $\operatorname{dim} \mathcal{N}(\lambda) \geq n$, the first statement in (4.20) follows. The second statement in (4.20) is then a direct consequence of (4.19).

We can now see that the rank of the limiting matrix radius $\boldsymbol{R}_{+}(\lambda)$ can never be zero, so that the 'limit point' behavior of $\left(\mathbf{S}_{\lambda}\right)$ should not be determined by the equality $\boldsymbol{R}_{+}(\lambda)=0$, as one would expect from the separated endpoints case in [1, Theorem 4.4]. The result in Theorem 4.6 then motivates the following definition.

Definition 4.7 Let $\lambda \in \mathbb{C} \backslash \mathbb{R}$. Under Hypothesis 4.2 , we say that system $\left(\mathbf{S}_{\lambda}\right)$ is in the limit point case if $\operatorname{dim} \mathcal{N}(\lambda)=3 n$, while system $\left(\mathbf{S}_{\lambda}\right)$ is in the limit circle case if $\operatorname{dim} \mathcal{N}(\lambda)=4 n$.

Combining Theorem 4.6 and Corollary 4.5 then yields the next result.

Corollary 4.8 Let $\lambda \in \mathbb{C} \backslash \mathbb{R}$ and suppose that Hypothesis 2.2 holds. Then system $\left(\mathbf{S}_{\lambda}\right)$ is in the limit point case, resp. limit circle case, if and only if system $\left(\mathrm{S}_{\lambda}\right)$ is in the limit point case, resp. limit circle case.

Remark 4.9 In the scalar case $n=1$, we have $\operatorname{rank} \boldsymbol{R}_{+}(\lambda) \in\{1,2\}$. In this situation, system $\left(\mathbf{S}_{\lambda}\right)$ is either in the limit point case (when $\operatorname{rank} \boldsymbol{R}_{+}(\lambda)=1$ ) or in the limit circle case (when 
$\left.\operatorname{rank} \boldsymbol{R}_{+}(\lambda)=2\right)$. This is known as the Weyl alternative, compare with [35, Theorem 8.27] and [1, Theorem 4.17 and Corollary 4.19].

Remark 4.10 The augmentation of system $\left(\mathrm{S}_{\lambda}\right)$ into double dimension is a known technique for studying the problems with jointly varying endpoints; see, e.g., [10-15]. The transformation introduced in this paper has the advantage that it uses the solutions $z$ or $Z$ of $\left(\mathrm{S}_{\lambda}\right)$ rather their components $(x, u)$ or $(X, U)$ as in the above references. This yields a direct connection between the original system $\left(S_{\lambda}\right)$ and the augmented system $\left(\mathbf{S}_{\lambda}\right)$. For example, the boundary conditions in $[15$, Section 6$]$ are of the form

$$
R_{1}\left(\begin{array}{c}
-x_{0} \\
x_{N+1}
\end{array}\right)+R_{2}\left(\begin{array}{c}
u_{0} \\
u_{N+1}
\end{array}\right)=0
$$

with certain $2 n \times 2 n$ matrices $R_{1}$ and $R_{2}$. One can see that the new approach via (1.5) is much easier and more transparent. The relationship between the transformation in the above mentioned references and the transformation, which is utilized in this section, is determined by the multiplication of the data (from one side or from both sides) by the $4 n \times 4 n$ matrix

$$
\mathcal{T}:=\left(\begin{array}{cccc}
-I & 0 & 0 & 0 \\
0 & 0 & I & 0 \\
0 & I & 0 & 0 \\
0 & 0 & 0 & I
\end{array}\right)=\mathcal{T}^{-1}, \quad \text { e.g., } \quad \mathcal{T}\left(\begin{array}{c}
-x_{0} \\
x_{N+1} \\
u_{0} \\
u_{N+1}
\end{array}\right)=\left(\begin{array}{c}
x_{0} \\
u_{0} \\
x_{N+1} \\
u_{N+1}
\end{array}\right)=\left(\begin{array}{c}
z_{0} \\
z_{N+1}
\end{array}\right)
$$

gives a direct connection between the boundary conditions (4.21) and (1.5).

\section{Conclusion}

In this paper we demonstrated that $2 n$-dimensional discrete symplectic eigenvalue problems with jointly varying endpoints (including the periodic and antiperiodic boundary conditions) can be studied by a transformation to separated endpoints in the dimension $4 n$. This transformation works under the strong Atkinson condition on the original system, which turns out to be the weak Atkinson condition on the augmented system (Corollaries 4.4 and 4.5). As one of the main results, we showed that for every system $\left(\mathrm{S}_{\lambda}\right)$ there is a naturally associated limiting Weyl disk $D_{+}(\lambda)$ consisting of complex $2 n \times 2 n$ matrices, whose center $P_{+}(\lambda)$ and matrix radius $R_{+}(\lambda)$ can be explicitly calculated by the formulas in (2.12). Moreover, we showed (Theorem 2.11 and Corollary 2.12) that the rank of the matrix radius $R_{+}(\lambda)$ is equal to the number of square summable solutions of $\left(\mathrm{S}_{\lambda}\right)$ and that it is always at least $n$. Thus, system $\left(\mathrm{S}_{\lambda}\right)$ is in the limit point case, resp. limit circle case, when the rank of $R_{+}(\lambda)$ is $n$, resp. $2 n$. In turn, the limit point or limit circle classification of the augmented system $\left(\mathbf{S}_{\lambda}\right)$ is determined by the numbers $3 n$ and $4 n$ (Definition 4.7 and Corollary 4.8). As such, this paper completes and extends the known Weyl-Titchmarsh theory of discrete symplectic systems. We are convinced that the methods developed in this paper will also be useful for the study of the corresponding continuous time linear Hamiltonian eigenvalue problems. 
Authors' contributions

The authors declare that they conducted all the research in this paper in equal parts. All the authors read and approved the final manuscript.

\section{Acknowledgements}

This research was supported by the Czech Science Foundation under grant P201/10/1032 and by the European Social Fund and the state budget of the Czech Republic under the project 'Employment of Newly Graduated Doctors of Science for Scientific Excellence' (registration number CZ.1.07/2.3.00/30.0009).

Received: 11 April 2013 Accepted: 9 July 2013 Published: 7 August 2013

\section{References}

1. Šimon Hilscher, R, Zemánek, P: Weyl-Titchmarsh theory for discrete symplectic systems with general linear dependence on spectral parameter. J. Differ. Equ. Appl. (2013). doi:10.1080/10236198.2013.813496

2. Bohner, M, Došlý, O, Kratz, W: An oscillation theorem for discrete eigenvalue problems. Rocky Mt. J. Math. 33(4), 1233-1260 (2003)

3. Bohner, M, Došlý, O, Kratz, W: Sturmian and spectral theory for discrete symplectic systems. Trans. Am. Math. Soc. 361(6), 3109-3123 (2009)

4. Bohner, M, Sun, S: Weyl-Titchmarsh theory for symplectic difference systems. Appl. Math. Comput. 216(10), 2855-2864 (2010)

5. Clark, S, Zemánek, P: On a Weyl-Titchmarsh theory for discrete symplectic systems on a half line. Appl. Math. Comput. 217(7), 2952-2976 (2010)

6. Shi, Y: Weyl-Titchmarsh theory for a class of discrete linear Hamiltonian systems. Linear Algebra Appl. 416, $452-519$ (2006)

7. Sun, H: On the limit-point case of singular linear Hamiltonian systems. Appl. Anal. 89(5), 663-675 (2010)

8. Šimon Hilscher, R, Zemánek, P: Weyl-Titchmarsh theory for time scale symplectic systems on half line. Abstr. Appl. Anal. 2011, Article ID 738520 (2011)

9. Atkinson, FV: Discrete and Continuous Boundary Problems. Academic Press, New York (1964)

10. Bohner, M: Linear Hamiltonian difference systems: disconjugacy and Jacobi-type conditions. J. Math. Anal. Appl. 199(3), 804-826 (1996)

11. Hilscher, R, Růžičková, V: Riccati inequality and other results for discrete symplectic systems. J. Math. Anal. Appl. 322(2), 1083-1098 (2006)

12. Hilscher, R, Růžičková, V: Implicit Riccati equations and quadratic functionals for discrete symplectic systems. Int. J. Differ. Equ. 1(1), 135-154 (2006)

13. Hilscher, R, Zeidan, V: Symplectic difference systems: variable stepsize discretization and discrete quadratic functionals. Linear Algebra Appl. 367, 67-104 (2003)

14. Kratz, W: Definiteness of quadratic functionals. Analysis 23(2), 163-183 (2003)

15. Šimon Hilscher, R, Zeidan, V: Oscillation theorems and Rayleigh principle for linear Hamiltonian and symplectic systems with general boundary conditions. Appl. Math. Comput. 218(17), 8309-8328 (2012)

16. Kelley, WG, Peterson, AC: Difference Equations: An Introduction with Applications. Academic Press, San Diego (1991)

17. Wang, Y, Shi, Y: Eigenvalues of second-order difference equations with periodic and antiperiodic boundary conditions. J. Math. Anal. Appl. 309(1), 56-69 (2005)

18. Teschl, G: Jacobi Operators and Completely Integrable Nonlinear Lattices. Mathematical Surveys and Monographs, vol. 72. Am. Math. Soc., Providence (2000)

19. Ahlbrandt, CD, Peterson, AC: Discrete Hamiltonian Systems: Difference Equations, Continued Fractions, and Riccati Equations. Kluwer Academic, Boston (1996)

20. Mingarelli, AB: A limit-point criterion for a three-term recurrence relation. C. R. Math. Acad. Sci. Soc. R. Can. 3(3), 171-175 (1981)

21. Šimon Hilscher, R, Zeidan, V: Symmetric three-term recurrence equations and their symplectic structure. Adv. Differ. Equ. 2010, Article ID 626942 (2010)

22. Ren, G, Shi, Y: Defect indices and definiteness conditions for a class of discrete linear Hamiltonian systems. Appl. Math. Comput. 218(7), 3414-3429 (2011)

23. Shi, Y: On the rank of the matrix radius of the limiting set for a singular linear Hamiltonian system. Linear Algebra Appl. 376, 109-123 (2004)

24. Shi, Y: Spectral theory of discrete linear Hamiltonian systems. J. Math. Anal. Appl. 289(2), 554-570 (2004)

25. Bohner, M: Discrete linear Hamiltonian eigenvalue problems. Comput. Math. Appl. 36(10-12), 179-192 (1998)

26. Šimon Hilscher, R, Zeidan, V: Rayleigh principle for time scale symplectic systems and applications. Electron. J. Qual. Theory Differ. Equ. 2011, 83 (2011) (electronic)

27. Shi, Y, Chen, S: Spectral theory of second-order vector difference equations. J. Math. Anal. Appl. 239(2), 195-212 (1999)

28. Šimon Hilscher, R: Eigenvalue theory for time scale symplectic systems depending nonlinearly on spectral parameter. Appl. Math. Comput. 219(6), 2839-2860 (2012)

29. Chen, J, Shi, Y: The limit circle and limit point criteria for second order linear difference equations. Comput. Math. Appl. 47(6-7), 967-976 (2004)

30. Clark, S: A spectral analysis for self-adjoint operators generated by a class of second order difference equations J. Math. Anal. Appl. 197(1), 267-285 (1996)

31. Hinton, DB, Lewis, RT: Spectral analysis of second order difference equations. J. Math. Anal. Appl. 63, 421-438 (1978)

32. Jirari, A: Second order Sturm-Liouville difference equations and orthogonal polynomials. Mem. Am. Math. Soc. 113, 542 (1995)

33. Sun, H, Shi, Y: Limit-point and limit-circle criteria for singular second-order linear difference equations with complex coefficients. Comput. Math. Appl. 52(3-4), 539-554 (2006)

34. Sun, $H$, Shi, Y: Eigenvalues of second-order difference equations with coupled boundary conditions. Linear Algebra Appl. 414(1), 361-372 (2006)

35. Weidmann, J: Linear Operators in Hilbert Spaces. Graduate Texts in Mathematics, vol. 68. Springer, Berlin (1980) 
doi:10.1186/1687-1847-2013-232

Cite this article as: Šimon Hilscher and Zemánek: Weyl disks and square summable solutions for discrete symplectic systems with jointly varying endpoints. Advances in Difference Equations 2013 2013:232.

Submit your manuscript to a SpringerOpen ${ }^{\circ}$ journal and benefit from:

- Convenient online submission

- Rigorous peer review

- Immediate publication on acceptance

Open access: articles freely available online

- High visibility within the field

- Retaining the copyright to your article

Submit your next manuscript at $\gg$ springeropen.com 